\title{
Analysis of LoRaWAN Uplink with Multiple Demodulating Paths and Capture Effect
}

\author{
René Brandborg Sørensen, Student Member, IEEE, Nasrin Razmi, \\ Jimmy Jessen Nielsen, Member, IEEE, Petar Popovski, Fellow, IEEE
}

\begin{abstract}
Low power wide area networks (LPWANs), such as the ones based on the LoRaWAN protocol, are seen as enablers of large number of IoT applications and services. In this work, we assess the scalability of LoRaWAN by analyzing the frame success probability (FSP) of a LoRa frame while taking into account the capture effect and the number of parallel demodulation paths of the receiving gateway. We have based our model on the commonly used SX1301 gateway chipset, which is capable of demodulating up to eight frames simultaneously; however, the results of the model can be generalized to architectures with arbitrary number of demodulation paths. We have also introduced and investigated three policies for Spreading Factor (SF) allocation. Each policy is evaluated in terms of coverage probability, FSP, and throughput. The overall conclusion is that the presence of multiple demodulation paths introduces a significant change in the analysis and performance of the LoRa random access schemes.
\end{abstract}

Index Terms-LoRa, LoRaWAN, frame success probability (FSP), Collision Timing, Capture-effect, SX1301.

\section{INTRODUCTION}

LoRaWAN is a popular low power wide area network (LPWAN) protocol. It is based on Semtechs' proprietary LoRa modulation, which uses a chirp spread spectrum (CSS) to enable long range and resilient transmissions. The access scheme of LoRaWAN resembles a pure Aloha protocol, however LoRaWAN provides several narrow-band channels and quasiorthogonal spreading factors (SF) to make LoRaWAN scalable. A considerable research interest has arisen recently about the obtainable link budget of LoRa transmissions, along with studies of capture effect, collisions, and the non-orthogonality of SFs in LoRa-based networks.

There are three mechanisms in LoRaWAN that determine the network performance: coverage, capture effect, and the demodulation capabilities of the receiver. All of these are affected by the SF allocation. The coverage probability is determined by the device location and the sensitivity of the SF. The capture probability is a function of the interference from transmissions using the same SF (co-SF interference) or different SFs (inter-SF interference). Finally the choice of the SF controls the tradeoff between the transmission robustness and collision probability. On one hand, the transmissions that use lower SFs are less robust to noise and interference, resulting in a worsened coverage. On the other hand, lower

This work has been supported by the European Research Council (ERC Consolidator Grant Nr. 648382 WILLOW) within the Horizon 2020 Program.

All authors are with the Department of Electronic Systems, Aalborg University, Denmark (Email: \{rbs,razmi,jjn,petarp\} @es.aau.dk).
SF results in a higher rate, which, for fixed payload, translates into a shorter packet duration. This implies that the probability to cause or experience collision (interference) for that packet is lowered for lower SFs. In addition, a transmission with a lower SF occupies a demodulation path in the demodulator for a shorter time interval compared to a transmission with a larger SF.

The initial research in LoRaWAN modeled the protocol as pure Aloha channels for each SF and channel pair as in [1]. This work neglects inter-SF interference and capture effect. The performance of LoRa has been analyzed and modeled for a single LoRaWAN cell using stochastic geometry and considering capture effect in [2]-[6]. The authors in [2] model the co-SF interference by considering the interference from the strongest co-SF interferer device. The results show that, the co-SF interference effect increases as the number of devices increases, such that the network becomes interference-limited. The authors in [3] provide a framework to evaluate only the coSF interference by considering the level of overlap between the interfering packets. Inter-SF interference is modeled with the Co-SF interference in [4]-[6]. The authors of [4] consider the allocation of SFs in order to maximize the average coverage probability by assigning SFs to devices based on their distance to the gateway and SF sensitivities. The scalability and throughput of LoRaWAN deployments have been evaluated in [5], [6] based on the capture effect and coverage models. Both papers verify that both co-SF and inter-SF transmissions have a considerable effect on the capture probability, and thereby on the LoRaWAN scalability.

A mechanism impacting frame reception beyond coverage and the capture effect was identified experimentally in [7]. The SX1276 LoRa transceiver is found to lock onto frames after detecting four symbols of a preamble. The reception will fail if another frame (even one that captures the channel) begins transmission before the first LoRa frame has been received or certain other timing conditions have been fulfilled.

In this paper, we evaluate the scalability of LoRaWAN analytically by developing a joint model for coverage, capture effect and demodulation capabilities for LoRa transmissions. We build and evaluate our model on the basis of a SX1301 gateway; however, the model and the methodology can be applied for any architecture. The SX1301 is capable of demodulating 8 frames, simultaneously, any transmissions beyond this will be dropped. To our knowledge, this practical limitation has not been included in the previous models from 
Fig. 1. LoRa Frame structure as described in [8]. LoRaWAN frames, consisting of header and payload, are transmitted as the payload of a LoRa frame.

the literature. Finally, we evaluate the performance of several SF allocation schemes based on the developed model.

The rest of paper is organized as follows: In Section II we explain the scenario, assumptions, conditions and parameters of our analysis. The analysis is contained within Section III Section IV presents the numerical results and the associated discussion. Finally, the paper is concluded in Section $\mathrm{V}$

\section{SCENARIO}

We consider a single LoRa channel with a bandwidth $B W=125[\mathrm{kHz}]$. Each device transmits LoRaWAN frames with payloads of size $B$ bytes at a rate $\lambda_{d}$. A device is assigned a fixed SF $m$ to be used for transmission, where $M=\{7,8, \ldots, 12\}$ and $m \in M$ as allowed in LoRaWAN [9]. The symbol period for a given $m$ is $T_{s_{m}}=\frac{2^{m}}{B W}$. A LoRA frame uses the LoRaWAN frame as a payload. Each LoRa frame contains a preamble of 10 to 65539 symbols (default is 12), a LoRa header, payload and, optionally, a CRC for the payload as depicted in Fig. 11 The number of symbols needed for transmission of the LoRa header and the LoRaWAN payload using SF $m$ can be found as (1) from [8].

$$
n_{p k t_{m}}=8+\max \left(\left\lceil\frac{(8 B-4 m+44)}{\left(4 m-2 \cdot \mathbb{I}_{\mathrm{DE}}\right)}\right\rceil \cdot(C R+4), 0\right),
$$

where $C R$ is the coding rate. $C R=i$ for $i \in\{1,2,3,4\}$ corresponds to a coding rate equal to $\frac{4}{4+i}$. The indicator $\mathbb{I}_{\mathrm{DE}}=1$ if low data rate optimization is used and $\mathbb{I}_{\mathrm{DE}}=0$ otherwise. Low data rate optimization increases robustness towards clock drift and is mandatory for SF $\{11,12\}$.

The total number of symbols transmitted for a complete frame with SF $m$ is $n_{f_{m}}=n_{\text {pre }}+n_{p k t_{m}}$ where $n_{\text {pre }}$ denotes the number of symbols in the preamble for $m$. Then, the total transmission time is $T_{f_{m}}=T_{s_{m}} \cdot n_{f_{m}}$.

\section{A. Deployment Model}

We consider $N$ devices distributed uniformly in a circular region with radius $R$ with a gateway at the center. The distribution of distance $r$ between the gateway and the devices is defined as $g_{R}(r)$

$$
g_{R}(r)= \begin{cases}\frac{2 r}{R^{2}} & 0 \leq r \leq R \\ 0 & r \geq R\end{cases}
$$

The aggregated arrival rate of all devices can be computed as $\lambda=N \cdot \lambda_{d}$ as long as $\lambda_{d}<\mu_{d}$ where $\mu_{d}$ is equal to $\frac{1}{T_{f_{m}} / D C}$ and $D C$ is the duty-cycle given as a fraction [10].

Our model is based on SX1301 gateways, which is in widespread use for outdoor LoRaWAN deployments. SX1301 is capable of detecting preambles for all SFs for up to 8 channels at a time. This discrepancy between detection and demodulation is due to the fact that the SX1301 architecture separates the preamble detection from the data acquisition [11], [12].

\section{B. Channel model}

All devices transmit with power equal to $P_{0}=14[\mathrm{dBm}]$. Let $A\left(f_{c}\right)$ be the deterministic part of the path loss model with carrier frequency $f_{c}$ and noise power $o_{n}^{2}=-174+10 \log (B W)$ $[\mathrm{dBm}]$, where the noise figure is aassumed to be $0[\mathrm{~dB}]$. With the assumed bandwidth, we get $A\left(f_{c}\right)=f_{c}^{2} \cdot 10^{-2.8}$. We define $c$ as in [6]

$$
c=\frac{P_{0} A\left(f_{c}\right)}{o_{n}^{2}}
$$

We denote the required receiver sensitivity for SF $m$ by $\theta_{R X_{m}}$ and let $\gamma_{m}^{(i)}, \gamma_{c o, m}^{(i)}$ and $\gamma_{i n t, m}^{(i)}$ denote the received signal to noise ratio (SNR), the signal to noise plus interference ratios (SINR) of only co-SF interference and only inter$\mathrm{SF}$ interferences for frame $i$, respectively. $\gamma_{c o, i n t, m}^{(i)}$ denotes the received SINR in the presence of co-SF and inter-SF interferences. $\gamma_{m}^{(i)}, \gamma_{c o, m}^{(i)}, \gamma_{i n t, m}^{(i)}$ and $\gamma_{c o, i n t, m}^{(i)}$ are defined as

$$
\begin{gathered}
\gamma_{m}^{(i)}=c\left|h_{i}\right|^{2} r_{i}^{-\alpha}, \\
\gamma_{c o, m}^{(i)}=\frac{\left|h_{i}\right|^{2} r_{i}^{-\alpha}}{\sum_{k \in k_{c o, m}}\left|h_{k}\right|^{2} r_{k}^{-\alpha}+\frac{1}{c}}, \\
\gamma_{i n t, m}^{(i)}=\frac{\left|h_{i}\right|^{2} r_{i}^{-\alpha}}{\sum_{k \in k_{i n t, m}}\left|h_{k}\right|^{2} r_{k}^{-\alpha}+\frac{1}{c}}, \\
\gamma_{c o, i n t, m}^{(i)}=\frac{\left|h_{i}\right|^{2} r_{i}^{-\alpha}}{\sum_{k \in k_{c o, m}}\left|h_{k}\right|^{2} r_{k}^{-\alpha}+\sum_{k \in k_{i n t, m}}\left|h_{k}\right|^{2} r_{k}^{-\alpha}+\frac{1}{c}},
\end{gathered}
$$

where $\alpha$ is the path loss exponent and $h$ is the channel coefficient, which is assumed to be Rayleigh distributed. $k_{c o, m}$ and $k_{i n t, m}$ denote the number of interferer users with the same $\mathrm{SF}$ and different SFs, respectively.

\section{Transmission success}

A transmission must capture the channel in order to be successfully received. The channel is captured if the signal passess the thresholds $\Gamma_{m}, \Gamma_{c o}$ and $\Gamma_{i n t, m}$ for capturing the channel with respect to noise, co-SF interference and inter-SF interference, respectively. The probabilities for these events happening are:

$$
\begin{gathered}
\operatorname{Pr}\left(\gamma_{m}^{(i)}>\Gamma_{m}\right), \\
\operatorname{Pr}\left(\gamma_{c o, \text { int }, m}^{(i)}>\max \left(\Gamma_{c o}, \Gamma_{i n t, m}\right)\right), \\
\operatorname{Pr}\left(\gamma_{c o, m}^{(i)}>\Gamma_{c o}\right), \\
\operatorname{Pr}\left(\gamma_{i n t, m}^{(i)}>\Gamma_{i n t, m}\right) .
\end{gathered}
$$

In the case where there is no inter-SF or co-SF transmission condition 2) reduces to 3) or 4), respectively. Table @ lists $\Gamma_{m}$ and $\Gamma_{i n t, m}$ for $m \in M$. Notice that $\Gamma_{c o}$ is $6[\mathrm{~dB}]$, which is always larger than $\Gamma_{i n t, m}$ so condition 2) simplifies to $\operatorname{Pr}\left(\gamma_{\text {co, int }, m}^{(i)}>\Gamma_{\text {co }}\right)$.

A transmission may be received by the gateway if all the conditions for capturing the channel are fulfilled. However, we must consider the demodulation capability of the receiver which affects the probability of successful reception. If all 


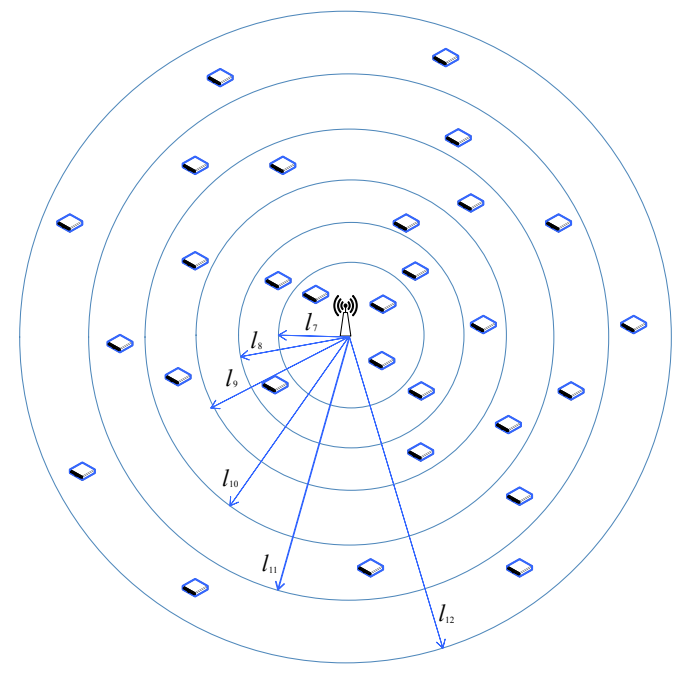

Fig. 2. SF allocation scheme based on annuli in a single gateway LoRaWAN cell.

demodulation paths are busy, then any additional detected frame is dropped. A demodulation path will be assigned to a frame after its preamble has been detected, i.e. four consecutive symbols of the preamble are detected. The header will then be demodulated and if it is correct, so will the rest of the frame. Therefore, the timing of the transmissions must be carefully accounted for in the model.

\section{SF Allocation}

SFs are allocated according to a scheme that is based on annuli, i.e. the radial distance of the device from the BS. Each annuli begins at the radial distance $l_{m-1}$ from the center of the cell and goes to $l_{m}$, such that $l_{12}=R$, as depicted in Fig. 2. Let $\delta_{m}$ denote the fraction of the device population assigned to $m$ and let $g_{m}(r)$ be the device density distribution for SF $m$. We define $\Delta_{X}$ as a set of mapping parameters $\Delta_{X}=\left\{\left\{\delta_{7}, \delta_{8}, \ldots, \delta_{12}\right\},\left\{l_{7}, l_{8}, \ldots, l_{12}\right\},\left\{g_{7}, g_{8}, \ldots, g_{12}\right\}\right\}$ for the $\mathrm{SF}$ allocation scheme $X$. We present three different allocation schemes and compute $\Delta$ in Sec. III-C

\section{UPLINK PERFORMANCE ANALYSIS}

In this section, we first analyze the FSP by taking into account the capture effect and the timing of collisions. Then, we investigate different SF allocation schemes.

A frame is received successfully if it captures the channel and is not dropped due to all demodulation paths being in use.

TABLE I

CHANNEL CAPTURE THRESHOLD PARAMETERS [6]

\begin{tabular}{lccc} 
SF & $\theta_{R X_{m}}[\mathrm{dBm}]$ & $\Gamma_{\boldsymbol{m}}[\mathrm{dB}]$ & $\Gamma_{i n t, \boldsymbol{m}}[\mathrm{dB}]$ \\
\hline 7 & -123 & -6 & -7.5 \\
8 & -126 & -9 & -9 \\
9 & -129 & -12 & -13.5 \\
10 & -132 & -15 & -15 \\
11 & -134.5 & -17.5 & -18 \\
12 & -137 & -20 & -22.5
\end{tabular}

To evaluate the uplink performance, we derive the probability of a LoRa frame from device $i$ being received successfully, which is denoted by $F S P_{m}^{(i)}$.

$$
F S P_{m}^{(i)}=F C P_{m}^{(i)} \cdot\left(1-F D P^{(i)}\right),
$$

where $F C P_{m}^{(i)}$ denotes the probability that the frame captures the channel. $F D P^{(i)}$ is the probability a frame being dropped due to all demodulation paths being busy.

To evaluate $F C P_{m}^{(i)}$ and $F D P^{(i)}$, we need to describe the number of packets which collide with the desired packet. Let $k_{c o, m}$ and $k_{i n t, m}$ denote the number of co-SF and inter$\mathrm{SF}$ frame transmissions, respectively, which interfere with the transmission of frame $i$ using SF $m$. Notice that we implicitly assume that all interfering frames overlap in time by evaluating the total number of interfering frames over a an entire frame. Realistically, several low SF frames could be placed non-overlapping times within the duration of a high SF frame. Therefore, we derive a lower bound on the $F C P_{m}^{(i)}$.

We define the traffic load for each source of co-SF and inter-SF interference loads over a period $\tau$ by $L_{c o, m}(\tau)$ and $L_{\text {int }, m}(\tau)$, respectively. When we evaluate the load over a period $\tau, L_{c o, m}(\tau)=\left(T_{f_{m}}+\tau\right) \cdot \lambda \cdot \delta_{m}$ such that we take into account interference from frame transmissions, which had begun before the start of the observed period $\tau$, but have not ended yet. By equivalent definition, $L_{i n t, m}(\tau)=$ $\sum_{p \in M, p \neq m}\left(T_{f_{p}}+\tau\right) \cdot \lambda \cdot \delta_{p}$ is the inter-SF load where $p$ denotes all SFs different from $m$. Omitting $\tau$ in the notation, the distributions of $k_{c o, m}$ and $k_{i n t, m}$ are given as:

$$
\begin{aligned}
P_{k_{c o, m}} & =\frac{\left(L_{c o, m}\right)^{k_{c o, m}} \cdot \exp \left(-L_{c o, m}\right)}{k_{c o, m} !} . \\
P_{k_{i n t, m}}= & \frac{\left(L_{i n t, m}\right)^{k_{i n t, m}} \cdot \exp \left(-L_{i n t, m}\right)}{k_{i n t, m} !} .
\end{aligned}
$$

\section{A. Derivation of $\mathrm{FCP}_{m}^{(i)}$}

To derive the probability of capturing the channel, we evaluate the 4 conditions for capture effect from Sec II-C

1) $k_{c o, m}+k_{i n t, m}=0$ : The probability of a single transmission being transmitted in the channel, is the probability of the devices in our deployment not generating a packet within two packet times.

$$
P_{\text {nocol }_{m}}=\exp \left(-\sum_{p \in M}\left(T_{f_{m}}+T_{f_{p}}\right) \cdot \delta_{p} \cdot \lambda\right) .
$$

Then the probability of successfully transmitting the frame is equal to

$$
P_{S_{\text {nocol }}^{(i)}}^{(i)}=P_{\text {nocol }_{m}} \cdot C P_{m}^{(i)},
$$

where the probability of capturing the channel is the coverage probability, $C P_{m}^{(i)}$, which can be determined as

$$
\begin{aligned}
& C P_{m}^{(i)}=\operatorname{Pr}\left(\gamma_{m}^{(i)}>\Gamma_{m}\right)=\operatorname{Pr}\left(c\left|h_{i}\right|^{2} r_{i}^{-\alpha} \geq \Gamma_{m}\right) \\
& =\int_{l_{m-1}}^{l_{m}} \exp \left(-\frac{\Gamma_{m} r_{i}^{\alpha}}{c}\right) g_{m}\left(r_{i}\right) d r_{i} .
\end{aligned}
$$

Eq. (17) can be derived easily based on $\left|h_{i}\right|^{2}$ being exponentially distributed under our Rayleigh assumption and the PDF of the population to $r_{i}$ being $g_{m}\left(r_{i}\right)$. 
2) $k_{\text {int }, m}=0:$ In the case that there is solely co-SF interference, we can express the probability of capturing the channel as

$$
\begin{gathered}
C P_{c o, m}^{(i)}\left(k_{c o, m}\right)=\operatorname{Pr}\left(\frac{\left|h_{i}\right|^{2} r_{i}^{-\alpha}}{\sum_{k=1}^{k_{c o, m}}\left|h_{k}\right|^{2} r_{k}^{-\alpha}+\frac{1}{c}} \geq \Gamma_{c o} \mid k_{c o, m}\right) \\
\int_{l_{m-1}}^{l_{m}} \exp \left(-\frac{\Gamma_{c o} r_{i}^{\alpha}}{c}\right) g_{m}\left(r_{i}\right)\left[I\left(r_{i}\right)\right]^{k_{c o, m}} d r_{i},
\end{gathered}
$$

where $I\left(r_{i}\right)$ is equal to

$$
I\left(r_{i}\right)=\int_{l_{m-1}}^{l_{m}} \frac{1}{1+\Gamma_{c o}\left(\frac{r_{i}}{r}\right)^{\alpha}} g_{m}(r) d r .
$$

3) $k_{c o, m}=0$ : In this case, there is no co-SF interference, $C P_{\text {int }, m}^{(i)}\left(k_{\text {int }, m}\right)$ can be expressed as

$$
\begin{gathered}
C P_{\text {int }, m}^{(i)}\left(k_{\text {int }, m}\right)=\operatorname{Pr}\left(\frac{\left|h_{i}\right|^{2} r_{i}^{-\alpha}}{\sum_{k=1}^{k_{\text {int }, m}}\left|h_{k}\right|^{2} r_{k}^{-\alpha}+\frac{1}{c}} \geq \Gamma_{\text {int }, m} \mid k_{\text {int }, m}\right) \\
\int_{l_{m-1}}^{l_{m}} \exp \left(-\frac{\Gamma_{\text {int }, m} r_{i}^{\alpha}}{c}\right) g_{m}\left(r_{i}\right)\left[\tilde{I}\left(r_{i}\right)\right]^{k_{i n t, m}} d r_{i},
\end{gathered}
$$

$\tilde{I}\left(r_{i}\right)$ is defined as

$$
\tilde{I}\left(r_{i}\right)=\int_{R \backslash R_{m}} \frac{1}{1+\Gamma_{i n t, m}\left(\frac{r_{i}}{r}\right)^{\alpha}} g_{p}(r) d r
$$

4) $k_{c o, m} \cdot k_{\text {int }, m} \neq 0:$ In case both co-SF and inter-SF interference are present. $C P_{i n t, m}^{(i)}\left(\left(k_{i n t, m}\right)\right)$ can be expressed as

$$
\begin{aligned}
& C P_{c o, i n t, m}^{(i)}\left(k_{c o, m}, k_{i n t, m}\right)= \\
& \operatorname{pr}\left(\frac{\left|h_{i}\right|^{2} r_{i}^{-\alpha}}{\sum_{k=1}^{k_{c o, m}}\left|h_{k}\right|^{2} r_{k}^{-\alpha}+\sum_{j=1}^{k_{i n t, m}}\left|h_{j}\right|^{2} r_{j}^{-\alpha}+\frac{1}{c}}\right. \\
& \left.\geq \max \left(\Gamma_{c o}, \Gamma_{i n t, m}\right) \mid k_{i n t, m}, k_{c o, m}\right) \\
& =\int_{l_{m-1}}^{l_{m}} \exp \left(-\frac{\Gamma_{c o} r_{i}^{\alpha}}{c}\right) g_{m}\left(r_{i}\right)\left[I\left(r_{i}\right)\right]^{k_{c o, m}}\left[I^{\prime}\left(r_{i}\right)\right]^{k_{i n t, m}} d r_{i},
\end{aligned}
$$

where $\max \left(\Gamma_{c o}, \Gamma_{i n t, m}\right)$ is always equal to $\Gamma_{c o} . I^{\prime}\left(r_{i}\right)$ is defined as

$$
I^{\prime}\left(r_{i}\right)=\int_{R \backslash R_{m}} \frac{1}{1+\Gamma_{c o}\left(\frac{r_{i}}{r}\right)^{\alpha}} g_{p}(r) d r .
$$

Then the probability of capturing the channel can be expressed as a weighed sum of the derived capture probabilities, where the weights are the probabilities of the particular capture scenario taking place.

$$
\begin{aligned}
& P_{\text {cap }_{m}}=P_{\text {nocol }_{m}} \cdot C P_{m}^{(i)} \\
& +\sum_{k_{c o, m}=1}^{\infty} P_{\left(k_{i n t, m}=0\right)} \cdot P_{k_{c o, m}} \cdot C P_{c o, m}^{(i)}\left(k_{c o, m}\right) \\
& +\sum_{k_{i n t, m}=1}^{\infty} P_{\left(k_{c o, m}=0\right)} \cdot P_{k_{i n t, m}} \cdot C P_{i n t, m}^{(i)}\left(k_{i n t, m}\right) \\
& +\sum_{k_{i n t, m}=1}^{\infty} \sum_{k_{c o, m}=1}^{\infty} P_{k_{c o, m}} \cdot P_{k_{i n t, m}} \cdot C P_{c o, i n t, m}^{(i)}\left(k_{c o, m}, k_{i n t, m}\right)
\end{aligned}
$$

Then, $F C P_{m}^{(i)}$ is given by

$$
F C P_{m}^{(i)}=P_{c a p_{m}}\left(\tau=T_{f_{m}}\right)
$$

\section{B. Calculation of $F D P^{(i)}$ and Throughput}

A SX1276 based LoRa receiver will 'lock onto' a frame once it has received 4 preambles as supported by [7], [8]. Preamble detection and frame demodulation are separated in the SX1301 and while it is capable of demodulating 8 frames simultaneously as supported by [11], [12], it is able to detect 48 preambles at once, i.e. a preamble for every SF and channel combination.

Since the preamble symbols and the 4 concurrent symbols needed for detection only constitute a small fraction of the total frame size, we approximate the capture probability as the coverage probability.

$$
C P_{\text {pre }_{m}}^{(i)} \approx C P_{m}^{(i)}
$$

We evaluate the FDP using the CDF of the Poisson distributed number of frames received by the demodulator at any given instant, $k_{M}=\sum_{m=7}^{12} k_{m}$ where $k_{m}$ denotes the number of frames being received using $m$ such that

$L_{M}=\sum_{m=7}^{12} L_{m}$ where $L_{m}=\lambda \cdot \delta_{m} \cdot T_{f_{m}} \cdot C P_{\text {pre }}^{(i)} \cdot(1-F D P)$

$$
F D P^{(i)}=1-\exp \left(-L_{M}\right) \cdot \sum_{k=0}^{7} \frac{\left(L_{M}\right)^{k}}{k !}
$$

Eq. 27) can be interpreted as the probability of there not being 7 or less concurrent frame receptions at the beginning of the reception of a new frame. $F S P_{m}^{(i)}$ is now computable as Eq. (12) by using Eq. (25) and Eq. (27).

The throughput of the cell can then be calculated as

$$
T_{\Delta_{\text {Scheme }}}=\sum_{m \in M} F S P_{m}^{(i)} \cdot \delta_{m} \cdot \lambda \cdot B
$$

where the throughput is defined as successfully received bytes of LoRaWAN payload per second.

\section{SF Allocation}

In this subsection, we compute $\delta_{m}, l_{m}$ and $g_{m}(r)$ for $m \in M$ for three SF allocation schemes. The cell center and edge for every scheme are defined as $l_{6}=0$ and $l_{12}=R$. All parameters for $\Delta$ which are presented in this subsection, can be found in Tab. [II

1) Uniform: In this scheme, the cell is not divided into annuli and instead SFs are assigned to devices uniformly. We denote the SF allocation set for this scheme by $\Delta_{U n i}$. Thus, $\delta_{m}=\frac{1}{6}$ and $g_{m}(r)=g_{R}(r)$. In this case, $l_{m}=R$ and $l_{m-1}=0$ for $m \in M$ is used in the model. 
TABLE II

SF ALLOCATION SCHEMES

\begin{tabular}{|c|c|c|c|c|c|}
\hline SF & $\Delta_{\text {Uniform }}$ & \multicolumn{2}{|c|}{$\Delta_{\text {Distance }}$} & \multicolumn{2}{|c|}{$\Delta_{\text {Eqload }}$} \\
\hline & $\delta_{m} l_{m}$ & & $l_{m}$ & $\delta_{m}$ & \\
\hline 7 & $1 / 6$ & .2 & .45 & .47 & .45 \\
\hline 8 & $1 / 6$ & .08 & .54 & .25 & .54 \\
\hline 9 & $1 / 6$ & .11 & .64 & .14 & .64 \\
\hline 10 & $1 / 6$ & .17 & .76 & .08 & .76 \\
\hline 11 & $1 / 6$ & .19 & .88 & .04 & .88 \\
\hline 12 & $1 / 6$ & .25 & 1 & .02 & 1 \\
\hline
\end{tabular}

2) Distance: We denote the SF allocation set for this scheme by $\Delta_{\text {Dist }}$. We compute the borders of each annulus based on the deterministic path loss and the receiver sensitivity such that $l_{m}=\left(\frac{P_{0} \cdot A\left(f_{c}\right)}{\theta_{R X_{m}}}\right)^{\frac{1}{\alpha}}$. Then, we calculate $\delta_{m}$ as a function of the area of a given annulus to the total cell area $\delta_{m}=\frac{N_{m}}{N}=\frac{A_{m}}{A} . N_{m}$ is the number of devices that are assigned SF $m$ and $A_{m}$ denotes the area of the annuli $m$. This means that $g_{m}(r)=\frac{g_{R}(r)}{\delta_{m}}$.

3) Equivalent load: We let the $l_{m}$ be the same as for $\Delta_{\text {Dist }}$. In this allocation scheme, denoted $\Delta_{\text {Eqload }}$, we calculate $T_{f_{m}}$ for each SF and assign SFs to keep the load equal for each SF such that $\delta_{m}=\frac{1 / T_{f_{m}}}{\sum_{s=M} 1 / T_{f_{s}}}$. We keep the device density uniform throughout each annulus, but we assume a higher device density in the annuli closer to the gateway such that $\sum_{m \in M}\left(\delta_{m} \mid l_{m}\right)=1$.

\section{RESULTS AND DISCUSSION}

We have evaluated CP, FCP, FDP and FSP analytically using Eq. (17), Eq. (25), Eq. (27) and Eq. (12), which are derived in Sec. IIII Furthermore, a Matlab-based simulation of the unacknowledged LoRaWAN uplink was implemented and results were simulated. In the simulation, the reception conditions 1-4 are evaluated at the symbol level, whereas the analytical approximation is evaluated at the frame-level due to (13) and (14). This results in the FCP approximation being lower-bound. SX1301 is capable of parallel reception on 8 LoRa channels, $1 \leq N_{c} \leq 8$. In this article we have assumed that the frequency of all $N_{c}$ channels is the same for the evaluation of FCP, which is a fair assumption since all LoRaWAN channels in the EU $863-870 \mathrm{MHz}$ ISM Band are fairly close in frequency. The channels are still considered orthogonal to each-other when evaluating the FCP. The FDP takes into account traffic on all channels as discerned LoRa frames compete for the same demodulation paths. The FCPs of SF $\{7,10,12\}$ are plotted in Fig. 3 for $\Delta_{\text {Dist }}$. Calculating the load contribution on the FDP for each SF $m, L_{m} / L_{M}$, we get .59 for SF 12, .1 for SF 10 and .015 for SF 7 . Although calculated for a specific allocation scheme, we can make some general assertions with regards to the impact of different SFs on the reception probability. The higher SFs take up demodulations paths for much longer time, which makes any transmission for SF $m \in M$ more likely to be dropped.

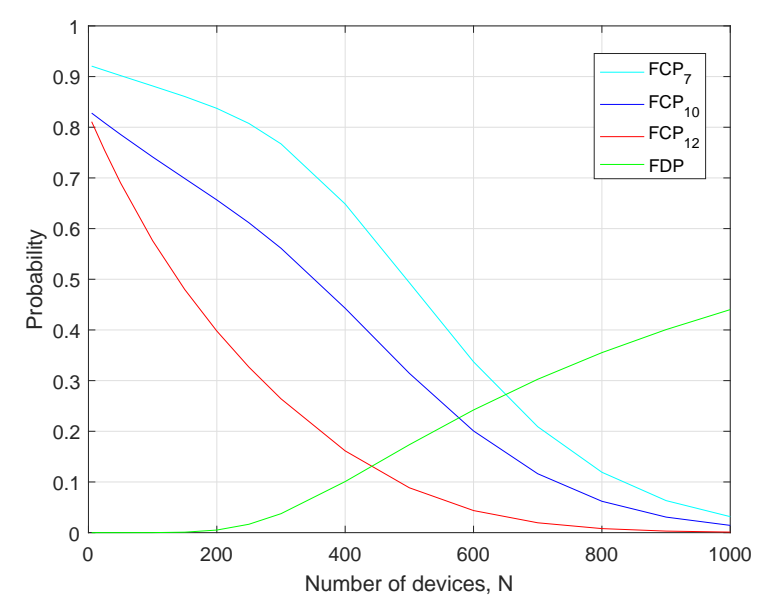

Fig. 3. Contribution of SFs 7, 10 and 12 on frame capture probability and frame drop probability for $\Delta_{D i s t}$. The traffic model considered was an mean inter-frame generation time of every tenth minute for each device and a payload size of 50 Bytes. This traffic model is assumed to be the same for the 8 channels, which are considered.

The FCP is also lower for the higher SF frames, which have a greater chance of experiencing interference. A higher coverage probability is seen for SF 7, which can be explained by SF 7 being allocated to devices in a punctured disc around the gateway.

In Fig. 4 and Fig. 5, we observe that the coverage probability for $\Delta_{\text {Dist }}$ and $\Delta_{\text {Eqload }}$ are .84 and .88 , respectively. The slight increase in coverage probability from $\Delta_{\text {Dist }}$ to $\Delta_{\text {Eqload }}$ can be explained by the allocation of more devices in the SF 7 annulus. It is also evident that the FDP has a non-negligible effect on the FSP especially when more devices are allocated to higher SFs, which is the case for $\Delta_{D i s t}$.

Results for the $C P, F C P_{m}^{(i)}$ and $F S P_{m}^{(i)}$ are not plotted for the uniform allocation scheme because the frame-based evaluation of interference results in a very low bound for $F C P_{m}^{(i)}$. This is due to the mixture of frames of both high and low SFs in the uniform allocation scheme.

The throughput of the three allocation schemes are depicted Fig. 6. While the $\Delta_{D i s t}$ provides better coverage than $\Delta_{U n i}$, it does not provide a much larger throughput on a cell basis since many devices are assigned to higher SFs. The equivalent-load allocation scheme assumes an uneven distribution of devices, which may not be the case often in actual deployments, but we see that the throughput is much larger in this case. This hints to cooperative assignment of SFs between gateways may possibly provide remarkable throughput improvements in LoRaWAN.

\section{CONClusion}

We have evaluated the uplink performance of a single gateway LoRaWAN deployment in terms of coverage, frame reception success probability and throughput. Collisions are evaluated in the time domain based on a traffic model in contrast to other works on capture effect in LoRaWAN, which evaluate capture effect for a fixed number of concurrent 


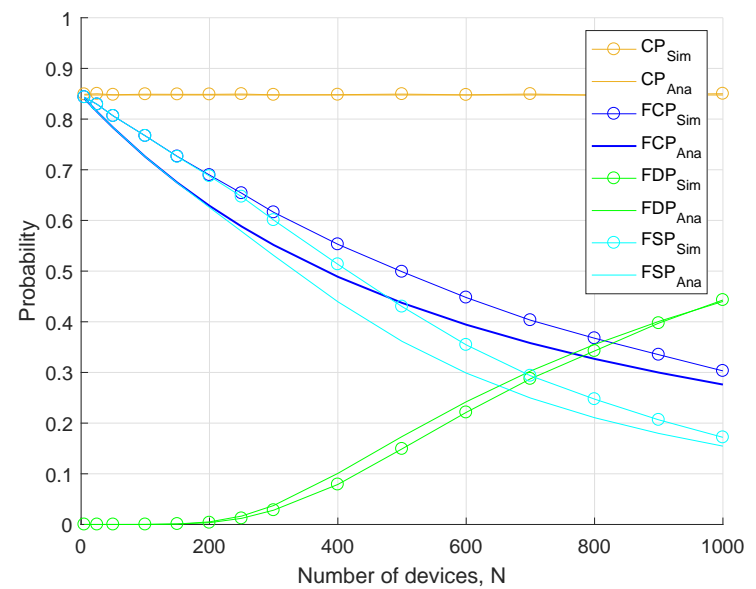

Fig. 4. Probabilities associated with frame capture for $\Delta_{D i s t}$. $\lambda_{d}=1 / 600, \mathrm{~B}=50$ and $N_{c}=8$.

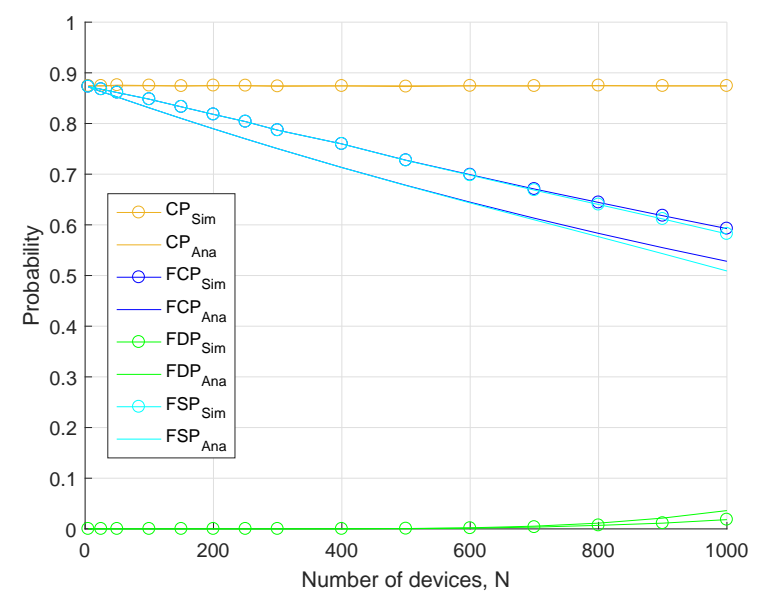

Fig. 5. Probabilities associated with frame capture for $\Delta_{\text {Eqload }}$. $\lambda_{d}=1 / 600, \mathrm{~B}=50$ and $N_{c}=8$.

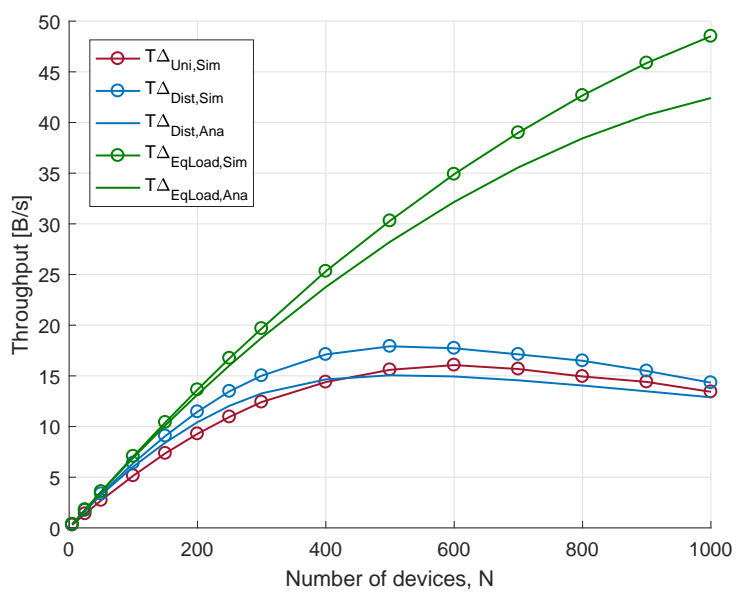

Fig. 6. Throughput for the LoRaWAN payload $[\mathrm{B} / \mathrm{s}]$.

$\lambda_{d}=1 / 600, \mathrm{~B}=50$ and $N_{c}=8$. transmissions. Unlike previous works, we have considered the demodulation capabilities of the gateway and specifically evaluated SX1301, although the model is applicable to any chipset. We showed that the demodulation capabilities of the receiver have a large impact on the probability of receiving frames successfully.

\section{REFERENCES}

[1] F. Adelantado, X. Vilajosana, P. Tuset-Peiro, B. Martinez, J. MeliaSegui, and T. Watteyne, "Understanding the limits of lorawan," IEEE Communications Magazine, vol. 55, no. 9, pp. 34-40, 2017.

[2] O. Georgiou and U. Raza, "Low power wide area network analysis: Can lora scale?" IEEE Wireless Communications Letters, vol. 6, no. 2, pp. 162-165, April 2017.

[3] Z. Li, S. Zozor, J. Drossier, N. Varsier, and Q. Lampin, "2d timefrequency interference modelling using stochastic geometry for performance evaluation in low-power wide-area networks," in 2017 IEEE International Conference on Communications (ICC), May 2017, pp. 17.

[4] J. Lim and Y. Han, "Spreading factor allocation for massive connectivity in lora systems," IEEE Communications Letters, vol. 22, no. 4, pp. 800803, April 2018.

[5] A. Mahmood, E. G. Sisinni, L. Guntupalli, R. Rondon, S. A. Hassan, and M. Gidlund, "Scalability analysis of a lora network under imperfect orthogonality," IEEE Transactions on Industrial Informatics, pp. 1-1, 2018.

[6] A. Waret, M. Kaneko, A. Guitton, and N. E. Rachkidy, "Lora throughput analysis with imperfect spreading factor orthogonality," IEEE Wireless Communications Letters, pp. 1-1, 2018.

[7] A. Rahmadhani and F. Kuipers, "When lorawan frames collide," Proc. of the 12th International Workshop on Wireless Network Testbeds. Experimental Evaluation \& Characterization (ACM WiNTECH 2018), 2018.

[8] Semtech, SX1276/77/78/79 Datasheet, rev. 5 ed., Aug. 2016.

[9] L. A. T. Committee, LoRaWAN Specification, v1.1 ed., LoRa Alliance, Oct. 2017.

[10] R. B. Sørensen, D. M. Kim, J. J. Nielsen, and P. Popovski, "Analysis of latency and mac-layer performance for class a lorawan," IEEE Wireless Communications Letters, vol. 6, no. 5, pp. 566-569, Oct 2017.

[11] Semtech, SX1301 Datasheet, v2.4 ed., June 2017.

[12] S. Co. (2017) Demodulation capacity of the sx1301. [Online]. Available: https://semtech.force.com/lora/articles/FAQ/Demodulation-capacity-of-the-SX1301. 